\title{
Serum potassium levels in Thai patients with T2DM and eGFR greater than or equal to $90 \mathrm{ml} / \mathrm{min} / 1.73 \mathrm{~m}^{2}$
}

\author{
Jirayut Janma ${ }^{1}$, Siribha Changsirikulchai ${ }^{*}$, Pornpen Sangthawan ${ }^{2}$, Nintita Sripaiboonkij Thokanit ${ }^{3}$, Suthee Rattanamongkolgul ${ }^{4}$ and $_{\text {Bandit }}$ \\ Thinkhamrop ${ }^{5}$
}

${ }^{1}$ Renal Division, Department of Medicine, Faculty of Medicine, Srinakharinwirot University, Thailand

${ }^{2}$ Renal Division, Department of Medicine, Faculty of Medicine, Prince of Songkla University, Thailand

${ }^{3}$ Ramathibodi Comprehensive Cancer Center, Faculty of Medicine, Ramathibodi Hospital, Mahidol University, Thailand

${ }^{4}$ Department of Preventive and Social Medicine, Faculty of Medicine, Srinakharinwirot University, Thailand

${ }^{5}$ Faculty of Public Health, Khon Kaen University, Thailand

\begin{abstract}
Background: Disturbances in potassium levels can occur in patients with renal dysfunction. This study's aim is to describe the serum potassium (sK) levels in Thai patients with type 2 Diabetes (T2DM) with or without hypertension (HT) and with estimated glomerular filtration rates (eGFR) greater than or equal to $90 \mathrm{ml}$ minute $/ 1.73 \mathrm{~m}^{2}$.

Methods: There was a cross sectional study performed throughout Thailand in 2016 for assessment on the quality of care among patients diagnosed with T2DM and HT. We selected a subgroup of patients who had data of sK levels and eGFR value greater than or equal to $90 \mathrm{ml} / \mathrm{minute} / 1.73 \mathrm{~m}^{2}$ for analyses.

Results: There were 3,719 subjects who met the inclusion criteria. The mean (SD) eGFR was 102.59 (9.12) $\mathrm{ml} / \mathrm{minute} / 1.73 \mathrm{~m}^{2}$. The mean (SD)sK level was 4.11 $(0.52) \mathrm{mEq} / \mathrm{L}$. The mean (SD) SBP and DBP were 130.86 (15.47) and 76.15 (10.14) $\mathrm{mmHg}$, respectively. The sK levels were categorized into 3 groups: low, normal and high at $<3.5,3.5-5.0$ and $>5 \mathrm{mEq} / \mathrm{L}$, respectively. The prevalence of hypokalemia was $6.7 \%$ and level less than $3 \mathrm{mEq} / \mathrm{L}$ was found in $55.6 \%$ of the hypokalemic population. The percentage of hypokalemia was found to be high in the group with diuretic usage. The prevalence of hyperkalemia was $2.9 \%$ and levels more than 5.5 $\mathrm{mEq} / \mathrm{L}$ was found in $21.7 \%$ of the hyperkalemic group.

Conclusions: The hypokalemia and hyperkalemia in T2DM with or without hypertension and eGFR $\geq 90 \mathrm{ml} / \mathrm{minute} / 1.73 \mathrm{~m}^{2}$ can be found. The sK levels less than 3 $\mathrm{mEq} / \mathrm{L}$ could be related to diuretic usage. Therefore, patients who are prescribed diuretics or renin angiotensin aldosterone blockades should have sK levels regularly monitored.
\end{abstract}

\begin{abstract}
Abbreviations: ACEI: Angiotensin converting enzyme inhibitors; ARBs: Angiotensin-receptor blockers; BMI: Body mass index; BP: Blood pressure; CKD: Chronic kidney disease; CKD-EPI: Chronic kidney disease epidemiology collaboration; DBP: Diastolic blood pressure; eGFR: Estimated glomerular filtration rates in $\mathrm{ml} /$ minute $/ 1.73$ $\mathrm{m}^{2}$; ESRD: End stage renal disease; HT: Hypertension; KDIGO: Kidney disease: Improving global outcomes; MedResNet: Medical Research Foundation; RAS: Renin-Angiotensin-Aldosterone system; SBP: Systolic blood pressure; SCr: Serum creatinine; Sk: Serum potassium; T2DM: Type 2 diabetes.
\end{abstract}

\section{Introduction}

The kidneys are primarily responsible for the function of regulating electrolytes including serum potassium (sK) [1]. The sK level is normally within the narrow range of 3.5 to $5.5 \mathrm{mEq} / \mathrm{L} \mathrm{[2].}$ The disorders of potassium homeostasis, particularly hyperkalemia, commonly occur in patients with chronic kidney disease (CKD) when the estimated glomerular filtration rate (eGFR) is less than $20 \%$ of normal and its prevalence is up to $50 \%$ in these patients [2-4]. Clinical complications of hyperkalemia are usually severe [3]. For hyperkalemia in diabetic patients, the mechanism of hyperkalemia is hyporeninemic hypoaldosteronism [5]. The hyperkalemia in this population may be provoked by clinical situations such as volume depletion and certain medications (e.g. nonsteroidalanti-inflammatory agents, and heparin) [5]. Previously, it has been postulated that hypokalemia is not a common disorder in CKD patients [6].

Recently published studies have shown that hypokalemia may be associated with the progression of renal disease [7-9]. It is also associated with morbidity, especially in patients with concurrent heart failure and CKD [7,9]. An observational study from Taiwan has shown that both hypokalemia and hyperkalemia are associated with an increased risk of end stage renal disease (ESRD) in the CKD population [9]. However, studies regarding sK levels in T2DM patients with early renal dysfunction or normal eGFR have not been clearly defined. The objective of this study is to assess sK levels in these populations and the prevalence of potassium disorders in this group.

${ }^{*}$ Correspondence to: Siribha Changsirikulchai, Renal Division, Department of Medicine, Faculty of Medicine Srinakharinwirot University, RangsitOnkharak, Nakhonnayok 26120, Thailand, Tel: 6637395085 Ext.10729, E-mail: siribha@swu.ac.th, siribha@g.swu.ac.th

Key words: type 2 diabetes, hyperkalemia, hypokalemia, chronic kidney disease, hypertension

Received: January 24, 2019; Accepted: February 11, 2019; Published: February 15,2019 


\section{Materials and methods}

\section{Study design}

This study is taken from a cross-sectional, multicenter survey "An assessment of quality of care among patients diagnosed with type 2 diabetes and hypertension visiting hospitals in the care of Ministry of Public Health and Bangkok Metropolitan Administration in Thailand". It was performed in Thailand in the year, 2015 by the Medical Research Foundation (MedResNet), Thailand. The dataset study protocols and case report forms are archived at the website http://www.damus.in.th/ damus/ [10].

\section{Study population}

T2DM subjects who were 20 years of age or older were recruited for this study. The inclusion criteria were; 1) patients with or without HT, with an eGFR $\geq 90 \mathrm{ml} /$ minute $/ 1.73 \mathrm{~m}^{2}$ as calculated with the Chronic Kidney Disease Epidemiology Collaboration (CKD-EPI) equation [11]. And 2) the sK levels and related variables had been obtained within the last 12 months. Figure 1 shows the study flow.

\section{Study variables and definitions}

Demographic data included in this study were age, gender, duration of T2DM, current use of ACEIs /ARBs or diuretics, blood pressure levels (BP) and body mass index (BMI). The most recent laboratory (defined by the last 12 months) results that we collected were HbAlc, serum creatinine $(\mathrm{sCr})$ and $\mathrm{sK}$ levels. The age groups were categorized as $<45,45-65$, and $>65$ years of age. The sK levels were categorized into 3 groups; $\mathrm{sK}<3.5 \mathrm{mEq} / \mathrm{L}$ as hypokalemia, $3.5-5 \mathrm{mEq} / \mathrm{L}$ as normokalemia and greater than $5 \mathrm{mEq} / \mathrm{L}$ as hyperkalemia [9].

\section{Definition of chronic kidney disease}

The eGFR was calculated using the CKD-EPI equation which was reported from the institutes' clinical care services. It was used to classify subjects according to the Kidney Disease: Improving Global Outcomes (KDIGO) CKD Work Group [12].

\section{The use of ACEIs, ARBs or diuretics}

Current use of ACEIs, ARBs or diuretics is defined as those that have been prescribed within the last 12 months.

\section{Statistical analyses}

The categorical variables are expressed as absolute numbers, percentages and proportions. Continuous variables are expressed as means with standard deviations. A chi-square test is used for independent comparisons of two categorical variables to determine if they are significantly related. Statistical tests were performed using Stata version 14.0 (StataCorp, College Station, TX).

\section{Results}

There were 11,043 patients with $\mathrm{eGFR} \geq 90 \mathrm{ml} /$ minute $/ 1.73 \mathrm{~m}^{2}$. Of these, a total of 3,719 cases having sK levels available for assessment were included in this study. The demographic characteristics of these subjects are presented in Table 1 . The majority of the population were female (2,560 cases; $68.84 \%)$. The mean age (SD) of the total number was $55.36(9.48)$ years. The mean (SD) systolic blood pressure (SBP) and diastolic blood pressure (DBP) were 130 (15.47) and 76 (10.14) $\mathrm{mmHg}$, respectively. The majority of this population had DM with HT (2,804, 75.40\%). Most of the patients with hypertension used ACEIs/ ARBs (2,260 cases, $80.60 \%)$. The mean (SD) eGFR was $102.59(9.12) \mathrm{ml} /$ minute/1.73 $\mathrm{m}^{2}$. The mean (SD) sK was $4.11(0.52) \mathrm{mEq} / \mathrm{L}$. The mean (SD) sK for hypokalemia, normokalemia and hyperkalemia was 3.18 (0.11), $4.14(0.07)$, and $5.56(0.59) \mathrm{mEq} / \mathrm{L}$, respectively. The prevalence of hypokalemia was $6.7 \%$ while the prevalence of hyperkalemia was $2.9 \%$ (Table1).

Regarding the severity of potassium disorders, we found that the majority of hypokalemic patients had mild hypokalemia (3-3.49 mEq/L) in $44.4 \%$ of the total cases. There were $35.6 \%$ and $20 \%$ of hypokalemic cases had sK $2.5-2.99 \mathrm{mEq} / \mathrm{L}$ and $<2.5 \mathrm{mEq} / \mathrm{L}$, respectively. In the hyperkalemic group, (78.3\%) mild hyperkalemia was commonly seen (sK $5-5.49 \mathrm{mEq} / \mathrm{L}$ ). There were only $21.7 \%$ that had $\mathrm{sK} \geq 5.5 \mathrm{mEq} / \mathrm{L}$.

In T2DM with HT group, we found that hypokalemia was common in cases with diuretic usage (13.2\%). On the other hand; in the ACEI/ ARB usage groups, only $2.6 \%$ exhibited hyperkalemia. The comparison of the hypokalemic group in T2DM with HT, we found that the number of hypokalemic patients was significant higher in diuretic use cases than in the non-use group $(\mathrm{p}<0.05)$ (Table 2$)$. The number of hypokalemic cases in ACEI/ARB use group was significantly lower than the non-use group. Regarding, hyperkalemic patients, we found that the number of hyperkalemic cases were significantly lower in ACEI/ARB usage group $(\mathrm{p}<0.05)$ (Table 2).

\section{Discussion}

Disorders of plasma potassium levels can be potentially lifethreatening conditions. The risk of ventricular arrhythmias and sudden cardiac arrest increases when sK levels are too low or too high [4].

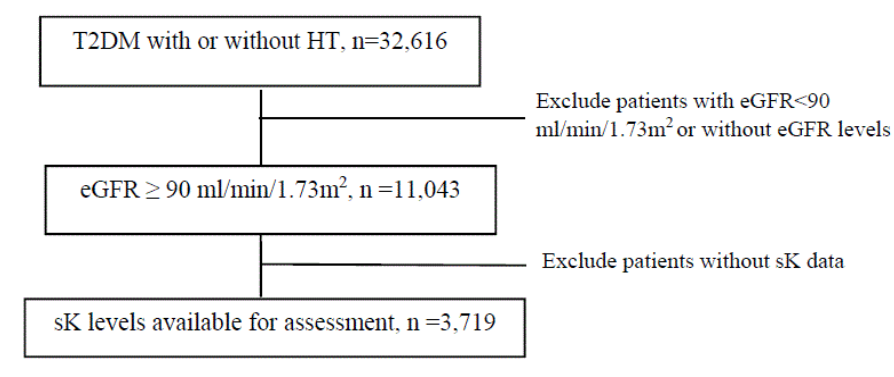

Figure 1. Flow of the enrolled subjects

Table 1. Baseline demographic and clinical characteristics

\begin{tabular}{|l|c|}
\hline Demographic data & Value \\
\hline Gender, female (n, \%) & $2,560(68.84 \%)$ \\
\hline DM with HT (n, \%) & $2,804(75.40 \%)$ \\
\hline ACEI/ ARB use (n, \%) & $2,260(80.60 \%)$ \\
\hline Diuretics use (n, \%) & $544(19.40 \%)$ \\
\hline Number of patients with sK (cases) & 3,719 \\
\hline sK<3.5 (n, \%) & $250(6.7 \%)$ \\
\hline sK 3.5-5 (n, \%) & $3,363(90.4 \%)$ \\
\hline sK $>5(\mathrm{n}, \%)$ & $106(2.9 \%)$ \\
\hline Mean age $($ year) $(\mathrm{SD})$ & $55.36(9.48)$ \\
\hline Mean eGFR $\left.(\mathrm{ml} / \mathrm{min} / 1.73 \mathrm{~m})^{2}\right)(\mathrm{SD})$ & $102.59(9.12)$ \\
\hline Mean of diabetic duration (years) (SD) & $6.04(0.05)$ \\
\hline Mean of HbA1C $(\%)(\mathrm{SD})$ & $8.13(2.03)$ \\
\hline Mean of SBP $(\mathrm{mmHg})(\mathrm{SD})$ & $130.86(15.47)$ \\
\hline Mean of DBP $(\mathrm{mmHg})(\mathrm{SD})$ & $76.15(10.14)$ \\
\hline Mean of BMI $\left(\mathrm{kg} / \mathrm{m}^{2}\right)(\mathrm{SD})$ & $26.51(5.10)$ \\
\hline Mean of sK levels $(\mathrm{mEq} / \mathrm{L})(\mathrm{SD})$ & $4.11(0.52)$ \\
\hline mean of sK<3.5 level (mEq/L) (SD) & $3.18(0.11)$ \\
\hline mean of sK 3.5-5 level (mEq/L) (SD) & $4.14(0.07)$ \\
\hline mean of sK $>5$ level (mEq/L) (SD) & $5.56(0.59)$ \\
\hline
\end{tabular}


Table 2. Serum potassium in patients with type 2 diabetes and hypertension

\begin{tabular}{|c|c|c|c|}
\hline \multirow{2}{*}{ Type of medications } & \multicolumn{3}{|c|}{ Potassium levels (sK) } \\
\cline { 2 - 4 } & $\mathrm{sK}<3.5(\mathrm{mEq} / \mathrm{L})$ & $\mathrm{sK} 3.5-5(\mathrm{mEq} / \mathrm{L})$ & $\mathrm{sK}>5(\mathrm{mEq} / \mathrm{L})$ \\
\hline \multicolumn{4}{|c|}{ Diuretic usage } \\
\hline Yes (n, \%) & $70(13.2 \%)^{*}$ & $446(84.2 \%)$ & $14(2.6 \%)$ \\
\hline No (n, \%) & $124(5.4 \%)$ & $2,080(91.5 \%)$ & $70(3.1 \%)$ \\
\hline \multicolumn{4}{|c|}{ ACEI/ARB usage } \\
\hline Yes (n, \%) & $134(6.3 \%)^{*}$ & $1,927(91.1 \%)$ & $55(2.6 \%)^{* *}$ \\
\hline No (n, \%) & $60(8.7 \%)$ & $599(87.1 \%)$ & $29(4.2 \%)$ \\
\hline
\end{tabular}

"Comparison hypokalemic and normokalemic group in type 2 diabetes and hypertension, $\mathrm{p}<0.05$

${ }^{* *}$ Comparison hyperkalemic and normokalemic group in type 2 diabetes and hypertension, $\mathrm{p}<0.05$

Both hyperkalemia and hypokalemia can occur in DM patients [13]. In diabetic patients who have a risk for hyperkalemia due to various mechanisms such as; reduced insulin levels leading to the accumulation of potassium in the extracellular space and hyporeninemic hypoaldosteronism $[13,14]$.

The increase of plasma tonicity in DM patients can contribute to hyperkalemia that results from the potassium shift from an intracellular to an extracellular space [15].

The other causes of hyperkalemia may be from multifactors in CKD patients for examples; use of potassium sparing diuretics, ACEI/ ARB agents and beta-blockers [4]. In our study, there is no data for potassium sparing diuretics and/or beta-blockers usage.

In T2DM patients, there is an impairment of insulin-mediated uptake of glucose, but the cellular uptake of potassium remains normal. Therefore, serum potassium is maintained within normal limits [15]. Consistent with our findings, the majority of cases have normal sK levels and a low prevalence of hyperkalemia regardless of ACEI/ARB usage. Mild hyperkalemia is predominating.

Our study has shown that the prevalence of hypokalemia in the early stages of CKD with T2DM, with or without hypertension, is greater than the prevalence of hyperkalemia. In contrast, many published studies have shown that the prevalence of hyperkalemia in diabetic patients is higher than those seen in the general population [4]. Hypokalemia is associated with ventricular arrhythmias, increased hospitalizations and greater mortality, particularly in CKD patients with chronic heart failure [7]. It also has been associated with hypertension and the progression of CKD [16]. Published literature has shown that even mild hypokalemia is associated with poor outcomes [7]. The explanation for hypokalemia in our study is that the Renin-Angiotensin-Aldosterone System (RAS) may be involved with the pathogenesis of hypokalemia in diabetic patients which was mainly due to the effects of diuretics [13].

The other mechanism of hypokalemia in the DM group is the hyperglycemic effect. The hyperglycemia has been linked to the transcription of angiotensinogen and the subsequent production of Angiotensin II from the local RAS [13].

It has also been linked to an increase in plasma renin activity, mean arterial pressure, and renal vascular resistance with the activation of circulating and local (intrarenal) RAS [13].

Also, the aldosterone enhances potassium secretion in the distal segments of the nephron which can contribute to the lowering of sK levels [1]. In addition, hypokalemia commonly occurs in the setting of gastrointestinal losses [16-18]. However, there is no data for gastrointestinal losses in our study which is limited by the study design.
Our study has shown the majority of the population as female $(68.84 \%)$ and of high age $(55.36 \pm 9.48$ years of age). There is a prior study that has found various etiologies of hypokalemia related to the female gender [19]. Kleinfeld et al reported that the predominance of hypokalemia in elderly groups was chiefly attributable to the female gender and was not dependent on diuretic usage. They concluded that it may be due to age and gender differences in body mass composition, which results in a lower sK in elderly females although, there is no direct evidence [19].

Our study has found that ACEI or ARB use can prevent hypokalemia in this population while prevalence of hyperkalemia was low. The explanation for low prevalence of hypokalemia in this group, the ACEI/ARB blocks aldosterone secretion and follows with inhibition of potassium secretion stimulation of K1 secretion [1]. We postulated that T2DM patients trend to have low sK as described above. Therefore, hypokalemia may be masked by the effects of ACEI/ARB. It confirmed by our postulation for low prevalence of hyperkalemia in ACEI/ARB group. The mechanisms of these findings need to be clarified. We conclude that the strategies to promote ACEI or ARB in DM with HT are recommended, in terms of blood pressure control and hypokalemia prevention.

There are some limitations in our study. The serum creatinine levels were not standardized with those used in the CKD-EPI formula. Our study found that there are 7,324 cases without sK data available. We postulated that this may be from lack of sK monitoring due to unforeseen circumstances. The further study should be done to determine these reasons.

\section{Conclusions}

In this study, potassium disorders, particularly hypokalemia, can be found in diabetic patients with a normal eGFR and prescribed diuretics. We encourage the monitoring of potassium levels in all patients regardless of normal renal function. Furthermore, ACEI/ARB agents can prevent hypokalemia in this group with a low prevalence of hyperkalemia.

\section{Declarations}

\section{Acknowledgement}

The authors would like to thank the Medical Research Foundation (MedResNet) for data set utilization. We thank Mr. Robert Cho for helping preparation with this article.

\section{Funding}

No funding support.

\section{Competing interests}

The authors declare that they have no competing interests.

\section{Ethics approval and consent to participate}

This is a secondary analysis from the DMHT dataset which was a cross-sectional survey done in Thailand by the researchers under the supervision of the Medical Research Foundation. This national survey was approved by the Institutional Ethical Review Committee for Research in Human Subjects, Thailand Ministry of Public Health, and the Royal Thai Army Medical Department Ethical Review Board. The secondary analysis of this study has been approved by the Institutional Ethical Review Committee for Research in Human Subjects, Srinakharinwirot University. We followed instructions of the Declaration of Helsinki, informed consent was not required. 


\section{Consent for publication}

Not applicable.

\section{Availability of data and material}

Dataset used in this study is the property of the Medical Research Foundation, therefore, it is not available to the public.

\section{Authors' contributions}

SC, JJ, and NT contributed to research idea, study design, data analysis and interpretation. PS contributed to interpretation. SR and BT provided data acquisition. SC, JJ and PS drafted the manuscript. All authors read and approved the final manuscript.

\section{References}

1. Palmer BF (2015) Regulation of potassium homeostasis. Clin J Am Soc Nephrol 10: 1050-1060. [Crossref]

2. Phillips SL, Polzin DJ (1998) Clinical disorders of potassium homeostasis: Hyperkalemia and hypokalemia. Vet Clin North Am Small Anim Pract 28: 545-564. [Crossref]

3. Einhorn LM, Zhan M, Hsu VD, Walker LD, Moen MF, et al. (2009) The frequency of hyperkalemia and its significance in chronic kidney disease. Arch Intern Med 169: 1156-1162. [Crossref]

4. Sarafidis PA, Blacklock R, Wood E, Rumjon A, Simmonds S, et al. (2012) Prevalence and factors associated with hyperkalemia in predialysis patients followed in a lowclearance clinic. Clin J Am Soc Nephrol 7: 1234-1241. [Crossref]

5. Uribarri J, Oh MS, Carroll HJ (1990) Hyperkalemia in diabetes mellitus. J Diabet Complications 4: 3-7. [Crossref]

6. Hayes J, Kalantar-Zadeh K, Lu JL, Turban S, Anderson JE, et al. (2012) Association of hypo- and hyperkalemia with disease progression and mortality in males with chronic kidney disease: The role of race. Nephron Clin Pract 120: c8-c16. [Crossref]

7. Bowling CB, Pitt B, Ahmed MI, Aban IB, Sanders PW, et al. (2010) Hypokalemia and outcomes in patients with chronic heart failure and chronic kidney disease: Findings from propensity-matched studies. Circ Heart Fail 3: 253-260. [Crossref]
8. Korgaonkar S, Tilea A, Gillespie BW, Kiser M, Eisele G, et al. (2010) Serum potassium and outcomes in CKD: Insights from the RRI-CKD cohort study. Clin J Am Soc Nephrol 5: 762-769. [Crossref]

9. Wang HH, Hung CC, Hwang DY, Kuo MC, Chiu YW, et al. (2013) Hypokalemia, its contributing factors and renal outcomes in patients with chronic kidney disease. PLoS One 8: e67140. [Crossref]

10. Sonthon P, Promthet S, Changsirikulchai S, Rangsin R, Thinkhamrop B, et al. (2017) The impact of the quality of care and other factors on progression of chronic kidney disease in Thai patients with Type 2 Diabetes Mellitus: A nationwide cohort study. PLoS One 12: e0180977. [Crossref]

11. Levey AS, Stevens LA, Schmid CH, Zhang YL, Castro AF 3rd, et al. (2009) A new equation to estimate glomerular filtration rate. Ann Intern Med 150: 604-612. [Crossref]

12. KDIGO (2012) Clinical practice guideline for the evaluation and management of chronic kidney disease (2013). Kidney International Supplements 3: 1-150.

13. Liamis G, Liberopoulos E, Barkas F, Elisaf M (2014) Diabetes mellitus and electrolyte disorders. World J Clin Cases 2: 488-496. [Crossref]

14. Lim HS, MacFadyen RJ, Lip GY (2004) Diabetes mellitus, the renin-angiotensinaldosterone system, and the heart. Arch Intern Med 164: 1737-1748. [Crossref]

15. Palmer BF, Clegg DJ (2015) Electrolyte and acid-base disturbances in patients with diabetes mellitus. $N$ Engl J Med 373: 548-559. [Crossref]

16. Greenlee M, Wingo CS, McDonough AA, Youn JH, Kone BC (2009) Narrative review: Evolving concepts in potassium homeostasis and hypokalemia. Ann Intern Med 150: 619-625. [Crossref]

17. Araki S, Haneda M, Koya D, Kondo K, Tanaka S, et al. (2015) Urinary potassium excretion and renal and cardiovascular complications in patients with type 2 diabetes and normal renal function. Clin J Am Soc Nephrol 10: 2152-2158. [Crossref]

18. Sharma S, McFann K, Chonchol M, de Boer IH, Kendrick J (2013) Association between dietary sodium and potassium intake with chronic kidney disease in US adults: A cross-sectional study. Am J Nephrol 37: 526-533. [Crossref]

19. Kleinfeld M, Borra S, Gavani S, Corcoran A (1993) Hypokalemia: Are elderly females more vulnerable? J Natl Med Assoc 85: 861-864. [Crossref]

Copyright: (C2019 Janma J. This is an open-access article distributed under the terms of the Creative Commons Attribution License, which permits unrestricted use, distribution, and reproduction in any medium, provided the original author and source are credited. 